\title{
INVESTIGAR: UNA COMPETENCIA INDISPENSABLE PARA EL PEDAGOGO
}

\author{
TO BE A RESEARCHER: A BASIC \\ COMPETENCE FOR THE PROFESIONAL \\ IN PEDAGOGY
}

Julián Ochoa García

Ligia García Béjar

Profesor Investigador, Escuela de Pedagogía, Universidad Panamericana, campus Guadalajara, México.

jochoa@up.edu.mx

ligarcia@up.edu.mx

\section{RESUMEN}

La formación de los estudiantes universitarios se ha vuelto cada vez más compleja. No se circunscribe sólo a la formación de especialistas en una de las ramas del conocimiento, también debe desarrollar las competencias transversales requeridas en todo egresado universitario. Entre las competencias que, de forma indispensable, debe adquirir un estudiante, se encuentran el desarrollo de habilidades informativas y de investigación. 
El concepto de alfabetización informacional ha sido estudiado ampliamente en los últimos años por varios especialistas, en especial en el campo de las ciencias de la información y la bibliotecología. Es esencial que alumnos y profesores sean autosuficientes para satisfacer sus necesidades de información al realizar investigaciones académicas fundamentadas que aporten contenidos al cúmulo de conocimientos ya existentes.

Los estudios a nivel universitario implican un trabajo de mayor profundidad y especialización por parte de los alumnos que los cursan, y especialmente en el tema de la investigación que deben realizar en el transcurso de los mismos. Es por eso que en el presente trabajo se profundizará en la importancia que guarda, en específico para los estudiantes de Pedagogía, el desarrollar correctamente las competencias informacionales, haciendo especial énfasis en las competencias en investigación.

Palabras clave: formación en competencias, alfabetización informacional, competencias en investigación, estudiantes universitarios.

\section{ABSTRACT}

Currently, the education of college students has become increasingly complex, because it is not limited to the training of, but to develop transversal skills required in all university graduates. Among the competencies that a student must acquire is the development of informative and research skills. The concept of information literacy has been studied extensively in recent years by several specialists, especially in the field of information science and librarianship. It is essential that students and teachers are self-sufficient to meet their information needs and to carry out well-founded academic research that contributes contents to the accumulation of existing knowledge.

Studies at the university level involve more in-depth work, especially on the subject of the research they must carry out during the course of their college education. In this essay we will be deepened in 
the importance it holds for students of Pedagogy to develop correctly the informational skills, with special emphasis on investigative skills.

Key words: skills education, informational literacy, research competences, college students.

\section{INTRODUCCIÓN}

Al hablar de la formación de los estudiantes, en especial de aquellos que tienen, en nuestro país, la fortuna de acceder a un programa de educación universitaria, es inevitable pensar en el tema de la investigación científica.

Aunque en muchas ocasiones la formación universitaria tiende a la educación profesionalizante - que prepara eficientemente y de una manera adecuada a los universitarios para enfrentarse ante los retos de la vida profesional-, no debe olvidarse la importancia que tiene el desarrollar las competencias adecuadas en el campo de la investigación científica en cada uno de los campos del conocimiento humano.

En relación con el tema de las competencias para la investigación, es de suma importancia el esclarecer claramente el concepto de investigación y, por otro lado, cuáles son las competencias necesarias para que un miembro de la comunidad universitaria cuente con las herramientas y habilidades necesarias para afrontar los retos que les presente la formación a ese nivel. Este trabajo pretende comprender el impacto que tiene, en el campo universitario, el ser competente en el área de la investigación científica, así como en la alfabetización informacional.

\section{LA «ALFABETIZACIÓN INFORMACIONAL» EN EL PEDAGOGO}

El concepto de «alfabetización informacional» ha sido estudiado ampliamente en los últimos años por varios especialistas, sobre todo en el campo de las ciencias de la información y la bibliotecología. El contexto natural en el cual se identifican las necesidades informativas 
de los miembros de una comunidad educativa es en el aula de clases; y es la figura del profesor quien deber ser uno de los principales facilitadores en el correcto manejo de la información académica, insumo básico en el proceso de formación de los estudiantes. Además, la competencia de investigación está impresa en la identidad del pedagogo, pues es un profesional llamado a crear estrategias, metodologías y recursos educativos, y a investigar los procesos educativos para su mejora.

El pedagogo está llamado a desarrollar un espíritu investigativo, acorde a tendencias actuales de la educación. No pueden existir elementos técnicos mejorables si un estudiante de pedagogía no se prepara para innovar procesos pedagógicos, capaces de generar transformaciones de la realidad educativa en cualquier entorno, que proporcionen soluciones a problemas registrados durante el desarrollo de la práctica educativa. En este artículo se identifica la vía de la alfabetización informacional del estudiante de pedagogía como medio para generar competencias de investigación que abonen a su perfil de egreso.

La alfabetización informacional (AI) es objeto de estudios recientes que analizan sus contenidos y alcances en el desarrollo de los individuos inmersos en la sociedad del conocimiento. De acuerdo con el nuevo documento de ACRL, Framework for Information Literacy for Higher Education, analizado por Alonso Arévalo (2016), se establece un nuevo concepto de ALFIN.

El concepto anterior era el siguiente: «AI es un conjunto de habilidades que requieren los individuos para reconocer cuándo se necesita información y tener la capacidad de localizar, evaluar y utilizar eficazmente la información requerida»(Alonso-Arévalo, 2016). De acuerdo con dicho concepto, el enfoque central de la AI se encontraba en el manejo de la información académica como insumo básico para el estudio y la investigación.

El nuevo concepto - más amplio y preciso, y al mismo tiempo más adecuado al perfil del pedagogo- afirma: 
AI es el conjunto de capacidades integradas que abarcan el descubrimiento reflexivo de la información, la comprensión y valoración de cómo se produce la información, el uso de la información en la creación de nuevos conocimientos y la participación ética en las comunidades de aprendizaje (Alonso A., 2016).

El concepto anterior se centra en la reflexión y las capacidades individuo que emplea la información y en el posible aporte del mismo a la comunidad en que se encuentra inmerso. Es decir, aquí el quid del asunto es la formación del sujeto en el mundo de la información, de manera que pueda aportar al mundo del conocimiento, después de analizar la información descubierta por él mismo. Nos referimos, nuevamente, al concepto de la formación para el mundo de la investigación académica, que a su vez puede tener muchas aristas de aplicación en el ámbito profesional.

Es pertinente mencionar el trabajo coordinado por Felicidad Campal, en el Grupo de Trabajo de Alfabetización Informacional (GTALFIN), el cual señala que:

[...] múltiples organismos nacionales e internacionales (OCDE, UNESCO, UE...) han hecho un esfuerzo en los últimos años por concretar cuáles deben ser las competencias clave en educación y, en lo que se refiere a la materia concreta de nuestro análisis, prácticamente hay unanimidad a la hora de señalar la necesidad de alfabetizar informacionalmente (Campal, F., 2016, p. 8).

Por tanto, ésta se enmarca como una competencia profesional básica para todo profesional.

Indica Campal en el mismo trabajo del GTALFIN que, como ejemplos de los trabajos de dichos organismos internacionales, se pueden citar los siguientes:

La OCDE, en relación a las habilidades y competencias del siglo XXI, contempla tres dimensiones en las que se pueden enseñar éstas: 
- Información (como fuente o como producto).

- Comunicación (las TIC fortalecen y aumentan las posibilidades de comunicación y colaboración en los miembros de una comunidad).

- Impacto ético-social (responsabilidad social e impacto social) (OCDE, 2010). La UNESCO unifica la Alfabetización Mediática con la Informacional (AMI) y las considera un conjunto combinado de competencias necesarias para sobrevivir en el siglo XXI. Está conformada por tres alfabetizaciones esenciales:

- Alfabetización en medios (Media Literacy).

- Alfabetización informacional (InformationLiteracy).

- Alfabetización digital (Digital Literacy).

La AMI abarca todos los tipos de medios de comunicación y otros proveedores de información como bibliotecas, archivos, museos o Internet (UNESCO, 2014) (p. 8).

Como se observa, el concepto de Alfabetización Informacional (AI) ha sufrido bastantes cambios y ajustes a los tiempos y necesidades actuales; y aunque existen diversos trabajos que realizan un buen acercamiento al concepto de AI, éste es un tema en constante adaptación a las situaciones tan cambiantes en la sociedad contemporánea.

A continuación, nos referiremos al tema de la investigación, así como a las competencias necesarias para un buen desempeño en dicho campo.

Tarea propia de las autoridades académicas es decidir sobre los mejores espacios y las mejores circunstancias para el desarrollo de estas competencias. Sin duda alguna, el aula presenta una situación ideal para formar progresivamente en estas habilidades, a lo largo del proceso de formación del alumno de pedagogía. En este sentido, otra acción esencial es asegurarse de que los profesores universitarios desarrollen estas competencias, o bien disponer de los medios para asegurarlo.

Un pedagogo que ha desarrollado competencias informacionales, mediáticas y digitales es un profesional capaz de argumentar, de reflexionar, está en un continuo estado de búsqueda, capacitado para construir conocimiento y modificar su entorno. 


\section{LAS COMPETENCIAS DE INVESTIGACIÓN EN EL PEDAGOGO}

La investigación para la práctica educativa es una oportunidad para aprender a desarrollar procesos sistemáticos en los que se puedan comprender significados sobre el entorno en el que se vive y se trabaja, observar, preguntar, registrar, interpretar, describir contextos y, por ende, proponer soluciones y perfeccionar la práctica educativa. El término «competencias» posee varias acepciones en los diversos trabajos académicos que se han enfocado a su estudio; para efecto del presente artículo se tomará el concepto de competencia acuñado por la American College of Occupational and Environmental Medicine, mencionado en el estudio realizado por Dulzaides y Molina (2007), por considerar que es uno de los más claros y precisos en el tema:

[las competencias representan] la posesión de calificaciones intelectuales, físicas y conductuales suficientes (conocimientos, habilidades y actitudes) para realizar una tarea o desempeñar un papel de manera adecuada para lograr un resultado deseado (p. 45).

Las competencias en investigación - también conocidas como «competencias investigativas»-, tienen su origen, en gran medida, en las propias derivadas de la Alfabetización Informacional (AI), pues su aporte en la formación académica de los estudiantes e investigadores actuales es de gran relevancia al buscar que los mismos adquieran los elementos necesarios para la autosuficiencia en materia informacional que, como ya se mencionó, son la materia prima o el insumo básico para lograr un nivel adecuado en el campo de la investigación científica.

Es indispensable hacer alusión nuevamente al nuevo concepto de Alfabetización Informacional de la ACRL, mencionado unos renglones arriba, debido a la relevancia del mismo en el campo de la formación para las competencias investigativas, al afirmar de manera contundente que:

AI es el conjunto de capacidades integradas que abarcan el descubrimiento reflexivo de la información, la comprensión y valoración de 
cómo se produce la información, el uso de la información en la creación de nuevos conocimientos y la participación ética en las comunidades de aprendizaje (Alonso-Arévalo, J., 2016).

En relación con el tema de la importancia de la investigación, Ruíz Ramírez afirma que:

[...] en el mundo actual, el verdadero ejercicio de la libertad y la soberanía está en el conocimiento; se necesita la ciencia para disminuir los límites de la ignorancia y aumentar la capacidad para resolver los problemas. Un mejor estándar de vida puede lograrse en un país que disponga de recursos humanos altamente adiestrados, formados en centros capaces de crear conocimientos y de formar profesionales imaginativos que puedan innovar y crear (Ruíz Ramírez, J., 2010).

Como se aprecia en la idea anteriormente descrita, no basta sólo con acceder a la información: debe contarse con las competencias adecuadas para saber qué hacer con los datos adquiridos para resolver una necesidad y, así, estar en condiciones de decidir adecuadamente al momento de enfrentarse al campo de la investigación.

Tal como lo mencionan Montes de Oca y Manchado, al seguir las ideas de Delors:

Entre las misiones y funciones reconocidas a la educación superior se encuentran: promover, generar y difundir conocimientos por medio de la investigación y, como parte de los servicios que ha de prestar a la comunidad, proporcionar la preparación técnica adecuada para contribuir al desarrollo cultural, social y económico de las sociedades, fomentando y desarrollando la investigación científica y tecnológica a la par que la investigación en el campo de las ciencias sociales, las humanidades y las artes creativas (Montes de Oca y Machado, 2009).

Afirman estos investigadores que «formar profesionales que utilicen los métodos de la ciencia para transformar la realidad es un imperativo, sin embargo su materialización en la práctica dista mucho 
de satisfacer las aspiraciones declaradas». Y no sólo eso, continúan señalando que:

[...] lograr dichas metas presupone una valorización mayor del componente o dimensión investigativa en todo el proceso de formación universitaria, así como una revisión del escenario de la formación y la necesidad de la interdisciplinariedad y transdisciplinariedad en el currículo, para concebir el desarrollo de habilidades investigativas como habilidades profesionales comunes de las diferentes carreras universitarias aunque, cada cual, con sus particularidades (Montes de Oca y Machado, 2009).

Continuando con las ideas del trabajo antes mencionado, se advierte que:

[...] apropiarse del método científico como objetivo educativo y por ende desarrollar habilidades investigativas en el pedagogo, no puede lograrse sólo a través de cursos dedicados a la metodología de la investigación, y si bien, a partir de algunas disciplinas «integradoras» y de determinadas estrategias curriculares, se alcanzan modos de actuación profesional vinculados con la dimensión científico técnica, es evidente que se deben aprovechar mucho más las potencialidades de todas y cada una de las disciplinas y asignaturas de manera que las habilidades investigativas verdaderamente se constituyan en modos de actuación de los futuros egresados, base esencial del desarrollo profesional (Montes de Oca y Machado, 2009).

El salón de clases, los espacios de prácticas, los proyectos interdisciplinares, etcétera, todo puede ayudar en la conformación de una «cultura de investigación, reflexión, transformación y sus implicaciones en la formación de profesionales de la educación comprometidos con mejores soluciones para el mundo dinámico de hoy» (Arias, 2018).

En relación con el tema de la formación de los investigadores, Roig Vila (2008) resalta la importancia que la educación ha tenido que afrontar múltiples desafíos a lo largo de la historia. Uno de los principales bien puede ser la creación y la transferencia de conocimiento. 
La información no es per se conocimiento, éste se basa en la información, pero se nutre, además, de no pocas dosis de raciocinio y, por qué no, de «racionamiento». Éste, sin duda, es un resultado secundario del desarrollo de competencias de investigación en el pedagogo.

La información ha constituido una materia prima esencial que se produce, se reelabora, se transforma y se comercializa [...]. A lo largo de la historia se produce, de hecho, una redefinición del concepto de cultura e, incluso, del de Educación —incluido, la educación universitaria- (Roig Villa, R., 2008).

Así, el autor antes mencionado concluye diciendo que:

[...] debemos afrontar el reto de desarrollar acciones continuadas tendientes a dar satisfacción, en términos de enseñanza permanente, innovación e investigación, pero también desarrollo y consolidación, al cambio acelerado que caracteriza la sociedad del conocimiento y de la comunicación. [...] (Lewis y Romiszowski, 1992; Cebrián de la Serna (coord.), 2000, citado por Roig Villa, R. 2008).

Volviendo al tema de la importancia de la investigación en el ámbito de la educación, Ruíz Ramírez nos recuerda que: «una de las funciones fundamentales de la Universidad es propiciar la generación de nuevos conocimientos mediante la investigación científica, tecnológica, humanística y social». Y continúa el mismo autor:

La investigación estimula el pensamiento crítico, la creatividad y es a través de ella, que el proceso de aprendizaje se vitaliza y se combate la memorización, que tanto ha contribuido a formar profesionales pasivos, pocos amantes de la innovación, con escasa curiosidad e iniciativa personal. La investigación es de importancia vital en los estudios de postgrado, no es posible tener egresados de alto nivel si no se investiga (Ruíz Ramírez, J., 2010).

Antes de pasar al siguiente tema, es oportuno recordar las palabras del profesor José López Yepes, en relación con la importancia de la investigación: 
«La investigación produce en el que la práctica una serie de efectos que proyectan las líneas de su configuración personal, de tener conciencia de estar desempeñando una tarea muy importante».

Y continúa el mismo autor recordando la importancia del componente personal en la investigación en los siguientes aspectos tomados de las ideas de Lasso de Vega: «Amor a la ciencia, curiosidad por lo desconocido y la satisfacción a causa de los nuevos descubrimientos» (Yepes, J., 1996, p. 40).

La formación en competencias de investigación en el pedagogo tiene algunas vertientes específicas que a continuación se describirán.

\subsection{Formación en investigación científica}

Ya se ha mencionado la importancia de formar a los nuevos investigadores en las competencias adecuadas para poder generar nuevo conocimiento y así aportar su «granito de arena» al cúmulo de información científica existente. Pero ¿cómo aportar a la formación en investigación científica?

Al respecto, Maldonado, L. y otros autores más (2007) explican que:

Los datos muestran que la investigación formativa se realiza a través de semilleros de investigación y la modalidad de trabajo de grado investigativo o proyectos de extensión a la comunidad. Sin embargo, existe poco acompañamiento por parte de los tutores, quizás porque no se tenía un tiempo especificado para la actividad investigativa (Maldonado, F. et. al., 2007).

Se puede detectar, entonces, la importancia que reviste la formación en el campo de la investigación científica para que los sujetos que se animen a trabajar en él, puedan hacerlo digna y propositivamente ante las necesidades que van surgiendo en la sociedad.

De acuerdo con lo anterior, la formación de dichas personas debe llevarse a cabo de una manera seria y disciplinada, pero a la vez 
conforme a la propuesta de soluciones aplicables a esos mismos requerimientos que se presentan en el contexto actual. En este sentido, Sánchez Puentes, en su libro titulado Enseñar a investigar: una didáctica nueva de la investigación de ciencias sociales y humanidades, subraya la relevancia que posee la formación en el campo de la investigación, pero dando especial énfasis a la misma como una «práctica concreta» (p. 11).

Se afirma en el mismo trabajo que «es más fecunda y da mejores resultados la enseñanza de la investigación que tiene como referente las prácticas concretas y los procesos efectivos manifestados durante la generación real de conocimiento» (Sánchez, R., 2014, p. 19).

La propuesta concreta de dicho trabajo parte de cuatro proposiciones específicas y que, de acuerdo con el autor, están estrechamente vinculadas:

Primera: es más prometedor enseñar a investigar teniendo como referente el proceso mismo de la generación efectiva de conocimiento científico. [...] Segunda: cambiar el énfasis de la didáctica de la investigación, pasando de una enseñanza teórica, abstracta y general a una didáctica práctica, basada en la capacitación y el entrenamiento. [...] Tercera: es conveniente planear estratégicamente la didáctica de la investigación científica a lo largo de los diferentes niveles de enseñanza. [...] Cuarta: el aprendizaje de la generación científica se optimiza al tener como aliado a otro investigador en plena producción (Sánchez, R., 2014, p. 20).

No es que nuestro autor menosprecie la formación teórica en el campo de la investigación científica; simplemente resalta la relevancia del asesor o tutor de los investigadores experimentados en la adquisición de las competencias necesarias en las nuevas promesas en dicha labor científica, mediante una nueva didáctica, distinta a la enseñanza tradicional en este campo. Puntualiza Sánchez:

El tutor no sólo enseña y trasmite saberes teóricos (know that) y saberes prácticos (know how), sino también debe trasmitir el significado y los valores últimos del quehacer científico (know why), el porqué o los «para qués», de carácter ético-político, de la generación científica (p. 17). 


\section{CONCLUSIONES}

En la actualidad, la educación universitaria del pedagogo debe implicar una formación integral, que considere los aspectos que preparen a los futuros profesionistas para afrontar los actuales retos sociales. Especial énfasis tiene la formación de los mismos en el campo de la investigación científica.

Para lograrlo es fundamental contar con cimientos adecuados que permitan, a quien se está formando, adquirir las competencias adecuadas para lograr este objetivo. La Alfabetización Informacional juega un papel preponderante en la adquisición de las competencias elementales para que los universitarios puedan transitar su etapa formativa con éxito.

Es esencial que los alumnos de pedagogía sean autosuficientes para satisfacer sus necesidades de información al realizar investigaciones académicas fundamentadas que aporten contenidos al cúmulo de conocimientos ya existentes, y que cuenten con las competencias necesarias para alcanzar su objetivo en los estudios en que se han matriculado.

Dentro de dichas competencias, juega un papel primordial la formación en las competencias en investigación. Así, los universitarios podrán acudir al conocimiento adquirido durante su etapa formativa para proponer soluciones ante las problemáticas concretas que se presentan en su vida profesional.

Papel fundamental juegan las autoridades académicas, los profesores y tutores universitarios. Ellos aportan no sólo el conocimiento teórico a sus alumnos (de vital importancia), sino también su experiencia profesional en el campo de la investigación; experiencia que puede ser transmitida, formando así nuevos semilleros de futuros investigadores, que cuenten con el respaldo de investigadores experimentados que les transmitan sus vivencias y sus experiencias al lado, claro está, de una sólida preparación teórica en el método científico. 


\section{BIBLIOGRAFÍA}

Arias, X. P. B., Castillo, L. C. Z., y Insuasty, E. A. (2017). El desarrollo de competencias investigativas de los docentes en formación en el contexto de la práctica pedagógica. Revista Folios, (47).

Alonso-Arévalo, J. (2016). Marco para la alfabetización informacional en educación superior (2016). Universo Abierto. Disponible en: https://universoabierto.com/2016/04/26/marco-para-la-alfabetizacion-informacional-en-educacion-superior /

Campal, Felicidad (coord.) (2016). Integración de las competencias ALFIN/AMI en el sistema educativo: referencias, contexto y propuestas [e-Book]. Grupo de Trabajo sobre Alfabetización Informacional del Consejo de Cooperación Bibliotecaria (GTALFIN). Madrid, Ministerio de Educación, Cultura y Deporte. Disponible en: http://www.ccbiblio.es/wp-content/uploads/Informe_GT_ALFIN.pdf

Comisión Técnica de Bibliotecas Escolares. 2011. Marco de referencia para las bibliotecas escolares. Ministerio de Educación. España. Disponible en: http:// bit.ly/24oqYjl

Dulzaides Iglesias, M. E.; Molina Gómez, A. (2007). La competencia informacional: concepción relevante a considerar en la Educación Superior. MediSur, 5, 44-47. Recuperado el 10 de septiembre de 2016, a partir de http:/ / www.redalyc.org/articulo. oa?id=180020178012

López Yepes, J. (1996). La aventura de la investigación científica. Guía del investigador y del director de investigación. Madrid. Editorial Síntesis. 
Maldonado, L. F.; Landazábal, D. P.; Hernández, J. C.; Ruíz, Y.; Claro, A.; Vanegas, H. y Cruz, S. (2007). Visibilidad y formación en investigación. Estrategias para el desarrollo de competencias investigativas. Studiositas, Vol. 2, No. 2, págs. 43-56. Disponible en: http: / / dialnet.unirioja.es/ servlet/ articulo?codigo=2719652

Roig Vila, R. (2008). De la investigación al conocimiento: reflexiones en torno a la innovación para la mejora del conocimiento educativo actual. Marfil. Disponible en: https:/ / goo.gl/QTZOR0

Ruíz Ramírez, J. (2010). Importancia de la investigación. Revista Científica, 20(2), 125-125. Disponible en: http:/ / www.scielo.org. ve/scielo.php?script=sci arttextypid=S0798-22592010000200001

Sánchez Puentes, R. (2014). Enseñar a Investigar: Una didáctica nueva de la Investigación en Ciencias Sociales y Humanidades. ISSUE. Universidad Nacional Autónoma de México. Disponible en: http://www.acuedi.org/ddata/11324.pdf 